\title{
Spectral properties of the logarithmic Laplacian
}

\author{
Ari Laptev ${ }^{1,2}(\mathbb{D}) \cdot$ Tobias Weth $^{3}$
}

Received: 29 September 2020 / Accepted: 18 March 2021 / Published online: 29 June 2021

(c) The Author(s) 2021

\section{Abstract}

We obtain spectral inequalities and asymptotic formulae for the discrete spectrum of the operator $\frac{1}{2} \log (-\Delta)$ in an open set $\Omega \in \mathbb{R}^{d}, d \geq 2$, of finite measure with Dirichlet boundary conditions. We also derive some results regarding lower bounds for the eigenvalue $\lambda_{1}(\Omega)$ and compare them with previously known inequalities.

\section{Introduction}

In the present paper, we study spectral estimates for the logarithmic Laplacian $L_{\Delta}=$ $\log (-\Delta)$, which is a (weakly) singular integral operator with Fourier symbol $2 \log |\eta|$ and arises as formal derivative $\left.\partial_{s}\right|_{s=0}(-\Delta)^{s}$ of fractional Laplacians at $s=0$. The study of $L_{\Delta}$ has been initiated recently in [5], where its relevance for the study of asymptotic spectral properties of the family of fractional Laplacians in the limit $s \rightarrow$ $0^{+}$has been discussed. In particular, it has been proved in [5, Theorem 1.5] that the principal Dirichlet eigenvalue of $L_{\Delta}$ in a bounded Lipschitz domain is given as a right derivative of principal Dirichlet eigenvalues of fractional Laplacians $(-\Delta)^{s}$ at $s=0$, whereas the corresponding principal eigenfunction arises as $L^{2}$-limit of corresponding Dirichlet eigenfunctions of $(-\Delta)^{s}$. Extensions of these results to higher eigenvalues and eigenfunctions were obtained afterwards in [8] together with uniform convergence

To V.G. Maz’ya with respect and admiration.

$凶$ Ari Laptev

a.laptev@imperial.ac.uk

Tobias Weth

weth@math.uni-frankfurt.de

1 Department of Mathematics, Imperial College London, Huxley Building, 180 Queen's Gate, London SW7 2AZ, UK

2 Sirius Mathematics Center Sirius University of Science and Technology, 1 Olympic Avenue, 354340 Sochi, Russia

3 Institut für Mathematik, Goethe-Universität, Frankfurt, Robert-Mayer-Straße 10, D-60629 Frankfurt, Germany 
and continuity results for these eigenfunctions. Further motivation for the study of $L_{\Delta}$ is given in [11], where it has been shown that this operator allows to characterize the $s$ dependence of solution to fractional Poisson problems for the full range of exponents $s \in(0,1)$. The logarithmic Laplacian also arises in the geometric context of the 0 -fractional perimeter, which has been studied recently in [6].

For matters of convenience, we state our results for the operator $\mathcal{H}=\frac{1}{2} L_{\Delta}$ which corresponds to the quadratic form

$$
\varphi \mapsto(\varphi, \varphi)_{\log }:=\frac{1}{(2 \pi)^{d}} \int_{\mathbb{R}^{d}} \log (|\xi|)|\widehat{\varphi}(\xi)|^{2} d \xi
$$

Here and in the following, we let $\widehat{\varphi}$ denote the Fourier transform

$$
\xi \mapsto \widehat{\varphi}(\xi)=\int_{\mathbb{R}^{d}} e^{-i x \xi} \varphi(x) d x
$$

of a function $\varphi \in L^{2}\left(\mathbb{R}^{d}\right)$. Let $\Omega \subset \mathbb{R}^{d}$ be an open set of finite measure, and let $\mathbb{H}(\Omega)$ denote the closure of $C_{c}^{\infty}(\Omega)$ with respect to the norm

$$
\varphi \mapsto\|\varphi\|_{*}^{2}:=\int_{\mathbb{R}^{d}} \log (e+|\xi|)|\widehat{\varphi}(\xi)|^{2} d \xi
$$

Then $(\cdot, \cdot)_{\log }$ defines a closed, symmetric and semibounded quadratic form with domain $\mathbb{H}(\Omega) \subset L^{2}(\Omega)$, see Sect. 2 below. Here and in the following, we identify $L^{2}(\Omega)$ with the space of functions $u \in L^{2}\left(\mathbb{R}^{d}\right)$ with $u \equiv 0$ on $\mathbb{R}^{d} \backslash \Omega$. Let

$$
\mathcal{H}: \mathcal{D}(\mathcal{H}) \subset L^{2}(\Omega) \rightarrow L^{2}(\Omega)
$$

be the unique self-adjoint operator associated with the quadratic form. The eigenvalue problem for $\mathcal{H}$ then writes as

$$
\left\{\begin{aligned}
\mathcal{H} \varphi & =\lambda \varphi, & & \text { in } \Omega, \\
\varphi & =0, & & \text { on } \quad \mathbb{R}^{d} \backslash \Omega .
\end{aligned}\right.
$$

We understand (1.3) in weak sense, i.e.

$$
\varphi \in \mathbb{H}(\Omega) \quad \text { and } \quad(\varphi, \psi)_{\log }=\lambda \int_{\Omega} \varphi(x) \psi(x) d x \text { for all } \psi \in \mathbb{H}(\Omega)
$$

As noted in [5, Theorem 1.4], there exists a sequence of eigenvalues

$$
\lambda_{1}(\Omega)<\lambda_{2}(\Omega) \leq \ldots, \quad \lim _{k \rightarrow \infty} \lambda_{k}(\Omega)=\infty
$$

and a corresponding complete orthonormal system of eigenfunctions. We note that the discreteness of the spectrum is a consequence of the fact that the embedding $\mathbb{H}(\Omega) \hookrightarrow L^{2}(\Omega)$ is compact. In the case of bounded open sets, the compactness of 
this embedding follows easily by Pego's criterion [18]. In the case of unbounded open sets of finite measure, the compactness can be deduced from [10, Theorem 1.2] and estimates for $\|\cdot\|_{*}$, see Corollary 2.3 below.

In Sect. 2, using the results from [5] and [7], we discuss properties of functions from $\mathbb{H}(\Omega)$. In particular, we show that $\left.e^{i x \xi}\right|_{x \in \Omega} \in \mathbb{H}(\Omega), \xi \in \mathbb{R}^{d}$, provided $\Omega$ is an open bounded sets with Lipschitz boundary.

In Sect. 3 we obtain a sharp upper bound for the Riesz means and for the number of eigenvalues $N(\lambda)$ of the operator $\mathcal{H}$ below $\lambda$. Here we use technique developed in papers $[3,4,13]$ and [14]. In [12] it was noticed that such technique could be applied for a class of pseudo-differential operators with Dirichlet boundary conditions in domains of finite measure without any requirements on the smoothness of the boundary.

We discuss lower bounds for $\lambda_{1}(\Omega)$ in Sect. 4. In Theorem 4.1 we present an estimate that is valid for arbitrary open sets of finite measure. For sets with Lipschitz boundaries, H.Chen and T.Weth [5] have proved a Faber-Krahn inequality for the operator $\mathcal{H}$ that reduces the problem to the estimate of $\lambda_{1}(B)$, where $B$ is a ball satisfying $|B|=|\Omega|$, see Corollary 4.3. In Theorem 4.4 we find an estimate for $\lambda_{1}\left(B_{d}\right)$, where $B_{d}$ is the unit ball, that is better in lower dimensions than the one obtained in Theorem 4.1. We also compare our results with bounds resulting from previously known spectral inequalities obtained in [1] and [2].

In Sect. 5 we obtain asymptotic lower bounds using the coherent states transformation approach given in [9]. It allows us to derive, in Sect. 6, asymptotics for the Riesz means of eigenvalues in Theorem 6.1 and for $N(\lambda)$ in Corollary 6.2. Here $\Omega \subset \mathbb{R}^{N}$ is an arbitrary open set of finite measure without any additional restrictions on the boundary.

Finally in Sect. 7 we obtain uniform bounds on the Riesz means of the eigenvalues using the fact that for bounded open sets with Lipschitz boundaries we have $\left.e^{i x \xi}\right|_{x \in \Omega} \in$ $\mathcal{D}(\mathcal{H})$.

We close this introduction with some remarks comparing the logarithmic Laplacian $\mathcal{H}=\frac{1}{2} L_{\Delta}$ with the spectral-theoretic $\log$ arithm $\log \left(-\Delta_{D}\right)$ of the Dirichlet Laplacian $-\Delta_{D}$ on a bounded Lipschitz domain $\Omega$ with form domain $H_{0}^{1}(\Omega)$. Clearly, the eigenvalues of $\log \left(-\Delta_{D}\right)$ are merely given as $\log \lambda_{k}^{D}(\Omega)$, where $\lambda_{k}^{D}(\Omega), k \in \mathbb{N}$ denote the Dirichlet eigenvalues of $-\Delta$ on $\Omega$. Comparing these eigenvalues with the eigenvalues $\lambda_{k}(\Omega)$ of $\mathcal{H}$, we note that

$$
\lambda_{k}(\Omega) \leq \frac{1}{2} \log \lambda_{k}^{D}(\Omega) \quad \text { for } \quad k \in \mathbb{N} .
$$

Indeed, this follows by combining [17, Theorem 5] with [8, Theorem 1.1(i)]. On the other hand, as we shall see in Corollary 6.2 below, the Weyl asymptotics of $\lambda_{k}(\Omega)$ as $k \rightarrow \infty$ are, up to first order, the same as those for $\frac{1}{2} \log \lambda_{k}^{D}(\Omega)$, the latter being a consequence of Weyl's classical result for the Dirichlet Laplacian. We also stress the obvious fact that the eigenfunctions of $\log \left(-\Delta_{D}\right)$ are the same as those of the Dirichlet Laplacian, while those of $\mathcal{H}=\frac{1}{2} L_{\Delta}$ differ significantly due to a much weaker boundary regularity. 


\section{Preliminaries and basic properties of eigenvalues}

As before, let $(\cdot, \cdot)_{\log }$ denote the quadratic form defined in (1.1), and let, for an open set $\Omega \subset \mathbb{R}^{d}, \mathbb{H}(\Omega)$ denote the closure of $C_{c}^{\infty}(\Omega)$ with respect to the norm $\|\cdot\|_{*}$ defined in (1.2).

Lemma 2.1 Let $\Omega \subset \mathbb{R}^{d}$ be an open set of finite measure. Then $(\cdot, \cdot)_{\log }$ defines a closed, symmetric and semibounded quadratic form with domain $\mathbb{H}(\Omega) \subset L^{2}(\Omega)$.

Proof Obviously, the form $(\cdot, \cdot)_{l o g}$ is symmetric. For functions $\varphi \in C_{c}^{\infty}(\Omega)$, we have

$$
(2 \pi)^{d}\|\varphi\|_{2}^{2}=\|\widehat{\varphi}\|_{2}^{2} \leq\|\varphi\|_{*}^{2} .
$$

Moreover, with $c_{1}:=\log (e+2)+\sup _{t \geq 2} \frac{\log (e+t)}{\log t}$ we have

$$
\begin{aligned}
\frac{\|\varphi\|_{*}^{2}}{c_{1}} & \leq\|\widehat{\varphi}\|_{2}^{2}+\int_{|\xi| \geq 2} \ln |\xi \| \widehat{\varphi}(\xi)|^{2} d \xi \\
& \leq(2 \pi)^{d}\left(\|\varphi\|_{2}^{2}+(\varphi, \varphi)_{\log }\right)-\int_{|\xi| \leq 2} \ln |\xi \| \widehat{\varphi}(\xi)|^{2} d \xi \\
& \leq(2 \pi)^{d}\left(\|\varphi\|_{2}^{2}+(\varphi, \varphi)_{\log }\right)+\|\ln |\cdot|\|_{L^{1}\left(B_{2}(0)\right)}\|\widehat{\varphi}\|_{\infty}^{2}
\end{aligned}
$$

while

$$
\|\widehat{\varphi}\|_{\infty}^{2} \leq\|\varphi\|_{1}^{2} \leq|\Omega|\|\varphi\|_{2}^{2}
$$

Consequently,

$$
\begin{aligned}
(\varphi, \varphi)_{l o g} & \geq \frac{\|\varphi\|_{*}^{2}}{(2 \pi)^{d} c_{1}}-\left(1+\frac{|\Omega|\|\ln |\cdot|\|_{L^{1}\left(B_{2}(0)\right)}}{(2 \pi)^{d}}\right)\|\varphi\|_{2}^{2} \\
& \geq\left(\frac{1}{c_{1}}-1-\frac{|\Omega|\|\ln |\cdot|\|_{L^{1}\left(B_{2}(0)\right)}}{(2 \pi)^{d}}\right)\|\varphi\|_{2}^{2} .
\end{aligned}
$$

In particular, $(\varphi, \varphi)_{\log }$ is semibounded. Moreover, it follows from (2.4) and the completeness of $\left(\mathbb{H}(\Omega),\|\cdot\|_{*}\right)$ that the form $(\varphi, \varphi)_{\text {log }}$ is closed on $\mathbb{H}(\Omega)$.

Lemma 2.2 Let $\Omega \subset \mathbb{R}^{d}$ be an open set of finite measure. Then

$$
\varphi \mapsto\|\varphi\|_{* *}^{2}:=\iint_{|x-y| \leq 1} \frac{(\varphi(x)-\varphi(y))^{2}}{|x-y|^{d}} d x d y
$$

defines an equivalent norm to the norm $\|\cdot\|_{*}$ defined in (1.2) on $C_{c}^{\infty}(\Omega)$.

Proof Let $\varphi \in C_{c}^{\infty}(\Omega)$. By [7, Lemma 2.7], we have

$$
\|\varphi\|_{2} \leq c_{2}\|\varphi\|_{* *} \quad \text { with a constant } \quad c_{2}>0 \text { independent of } \varphi \text {. }
$$


In particular, $\|\cdot\|_{* *}$ defines a norm on $C_{c}^{\infty}(\Omega)$. Next we note that, by [5, Theorem 1.1(ii) and Eq. (3.1)],

$$
(\varphi, \varphi)_{\log }=\frac{1}{2} \int_{\mathbb{R}^{d}}\left[L_{\Delta} \varphi(x)\right] \varphi(x) d x=\kappa_{d}\|\varphi\|_{* *}^{2}-\int_{\mathbb{R}^{d}}[j * \varphi] \varphi d x+\zeta_{d}\|\varphi\|_{2}^{2}
$$

with

$$
\kappa_{d}:=\frac{\pi^{-\frac{d}{2}} \Gamma(d / 2)}{4}, \quad \zeta_{d}:=\log 2+\frac{1}{2}(\psi(d / 2)-\gamma)
$$

and

$$
j: \mathbb{R}^{d} \backslash\{0\} \rightarrow \mathbb{R}, \quad j(z)=2 \kappa_{d} 1_{\mathbb{R}^{d} \backslash B_{d}}(z)|z|^{-d} .
$$

Here $\psi:=\frac{\Gamma^{\prime}}{\Gamma}$ is the Digamma function and $\gamma=-\Gamma^{\prime}(1)$ is the Euler-Mascheroni constant. Consequently, we have

$$
\begin{aligned}
\left|(\varphi, \varphi)_{\log }-\kappa_{d}\|\varphi\|_{* *}^{2}\right| & \leq\|j\|_{\infty}\|\varphi\|_{1}^{2}+\zeta_{d}\|\varphi\|_{2}^{2} \\
& \leq\left(\|j\|_{\infty}|\Omega|+\zeta_{d}\right)\|\varphi\|_{2}^{2} .
\end{aligned}
$$

As a consequence of (2.1) and (2.8), we find that

$$
\begin{aligned}
\|\varphi\|_{* *}^{2} & \leq \frac{1}{\kappa_{d}}\left[(\varphi, \varphi)_{\log }+\left(\|j\|_{\infty}|\Omega|+\zeta_{d}\right)\|\varphi\|_{2}^{2}\right] \\
& \leq \frac{1}{(2 \pi)^{d} \kappa_{d}}\left(1+\|j\|_{\infty}|\Omega|+\zeta_{d}\right)\|\varphi\|_{*}^{2} .
\end{aligned}
$$

Moreover, by (2.2), (2.3), (2.6) and (2.8) we have

$$
\begin{aligned}
& \frac{\|\varphi\|_{*}^{2}}{c_{1}} \leq(2 \pi)^{d}\left(\|\varphi\|_{2}^{2}+(\varphi, \varphi)_{l o g}\right)+\|\ln |\cdot|\|_{L^{1}\left(B_{2}(0)\right)}|\Omega|\|\varphi\|_{2}^{2} \\
& \quad \leq(2 \pi)^{d}\left(\kappa_{d}\|\varphi\|_{* *}^{2}+\left(1+\|j\|_{\infty}|\Omega|+\zeta_{d}\right)\|\varphi\|_{2}^{2}\right)+\|\ln |\cdot|\|_{L^{1}\left(B_{2}(0)\right)}|\Omega|\|\varphi\|_{2}^{2} \\
& \quad \leq c_{3}\|\varphi\|_{* *}^{2}
\end{aligned}
$$

with $c_{3}=(2 \pi)^{d} \kappa_{d}+c_{2}\left[(2 \pi)^{d}\left(1+\|j\|_{\infty}|\Omega|+\zeta_{d}\right)+\|\ln |\cdot|\|_{L^{1}\left(B_{2}(0)\right)}|\Omega|\right]$. Hence the norms $\|\cdot\|_{*}$ and $\|\cdot\|_{* *}$ are equivalent on $C_{c}^{\infty}(\Omega)$.

Corollary 2.3 Let $\Omega \subset \mathbb{R}^{d}$ be an open set of finite measure. Then the embedding $\mathbb{H}(\Omega) \hookrightarrow L^{2}(\Omega)$ is compact.

Proof Let $\tilde{\mathbb{H}}(\Omega)$ be defined as the space of functions $\varphi \in L^{2}\left(\mathbb{R}^{d}\right)$ with $\varphi \equiv 0$ on $\mathbb{R}^{d} \backslash \Omega$ and

$$
\iint_{|x-y| \leq 1} \frac{(\varphi(x)-\varphi(y))^{2}}{|x-y|^{d}} d x d y<\infty
$$


By [10, Theorem 1.2], the Hilbert space $\left(\tilde{\mathbb{H}}(\Omega),\|\cdot\|_{* *}\right)$ is compactly embedded in $L^{2}(\Omega)$. Since, by Lemma 2.2 , the norms $\|\cdot\|_{*}$ and $\|\cdot\|_{* *}$ are equivalent on $C_{c}^{\infty}(\Omega)$, the space $\mathbb{H}(\Omega)$ is embedded in $\tilde{\mathbb{H}}(\Omega)$. Hence the claim follows.

Corollary 2.4 Let $\Omega \subset \mathbb{R}^{d}$ be a bounded open set with Lipschitz boundary.

(i) The space $\mathbb{H}(\Omega)$ is equivalently given as the set of functions $\varphi \in L^{2}\left(\mathbb{R}^{d}\right)$ with $\varphi \equiv 0$ on $\mathbb{R}^{d} \backslash \Omega$ and

$$
\iint_{|x-y| \leq 1} \frac{(\varphi(x)-\varphi(y))^{2}}{|x-y|^{d}} d x d y<\infty
$$

(ii) $\mathbb{H}(\Omega)$ contains the characteristic function $1_{\Omega}$ of $\Omega$ and also the restrictions of exponentials $x \mapsto 1_{\Omega}(x) e^{i x \xi}, \xi \in \mathbb{R}^{d}$.

Proof (i) Let, as in the proof of Corollary 2.3, $\tilde{\mathbb{H}}(\Omega)$ be the space of functions $\varphi \in$ $L^{2}\left(\mathbb{R}^{d}\right)$ with $\varphi \equiv 0$ on $\mathbb{R}^{d} \backslash \Omega$ and with (2.9), endowed with the norm $\|\cdot\|_{* *}$. Since $\Omega \subset \mathbb{R}^{d}$ be a bounded open set with Lipschitz boundary, it follows from [5, Theorem 3.1] that $C_{0}^{\infty}(\Omega) \subset \tilde{\mathbb{H}}(\Omega)$ is dense. Hence the claim follows from Lemma 2.2.

(ii) follows from (i) and a straightforward computation.

Next we note an observation regarding the scaling properties of the eigenvalues $\lambda_{k}(\Omega)$.

Lemma 2.5 Let $\Omega \subset \mathbb{R}^{d}$ be a bounded open set with Lipschitz boundary, and let

$$
R \Omega:=\{R x: x \in \Omega\}
$$

Then we have

$$
\lambda_{k}(R \Omega)=\lambda_{k}(\Omega)-\log R \quad \text { for all } k \in \mathbb{N} .
$$

Proof Since $C_{0}^{\infty}(\Omega) \subset \mathbb{H}(\Omega)$ is dense, it suffices to note that

$$
\left(\varphi_{R}, \varphi_{R}\right)_{\log }=(\varphi, \varphi)_{\log }-\log R\|\varphi\|_{L^{2}\left(\mathbb{R}^{d}\right)}^{2} \quad \text { for } \quad \varphi \in C_{c}^{\infty}\left(\mathbb{R}^{d}\right)
$$

with $\varphi_{R} \in C_{c}^{\infty}\left(\mathbb{R}^{d}\right)$ defined by $\varphi_{R}(x)=R^{-\frac{d}{2}} \varphi\left(\frac{x}{R}\right)$, whereas $\left\|\varphi_{R}\right\|_{L^{2}\left(\mathbb{R}^{d}\right)}=$ $\|\varphi\|_{L^{2}\left(\mathbb{R}^{d}\right)}$. Since

$$
\widehat{\varphi R}=R^{\frac{d}{2}} \widehat{\varphi}(R \cdot)
$$


we have

$$
\begin{aligned}
& \left(\varphi_{R}, \varphi_{R}\right)_{\log } \\
& \quad=\frac{1}{(2 \pi)^{d}} \int_{\mathbb{R}^{d}} \log (|\xi|)|\widehat{\varphi R}(\xi)|^{2} d \xi=\frac{R^{d}}{(2 \pi)^{d}} \int_{\mathbb{R}^{d}} \log (|\xi|)|\widehat{\varphi}(R \xi)|^{2} d \xi \\
& =\frac{1}{(2 \pi)^{d}} \int_{\mathbb{R}^{d}}(\log (|\xi|)-\log R)|\widehat{\varphi}(\xi)|^{2} d \xi=(\varphi, \varphi)_{\log }-\log R\|\varphi\|_{L^{2}\left(\mathbb{R}^{d}\right)}^{2}
\end{aligned}
$$

as stated in (2.10).

\section{An upper trace bound}

Throughout this section, we let $\Omega \subset \mathbb{R}^{d}$ denote an open set of finite measure. Let $\left\{\varphi_{k}\right\}$ and $\left\{\lambda_{k}\right\}$ be the orthonormal in $L^{2}(\Omega)$ system of eigenfunctions and the eigenvalues of the operator $\mathcal{H}$ respectively. In what follows we denote

$$
(\lambda-t)_{+}= \begin{cases}\lambda-t, & \text { if } t<\lambda \\ 0, & \text { if } t \geq \lambda\end{cases}
$$

Then we have

Theorem 3.1 For the eigenvalues of the problem (1.3) and any $\lambda \in \mathbb{R}$ we have

$$
\sum_{k}\left(\lambda-\lambda_{k}\right)_{+} \leq \frac{1}{(2 \pi)^{d}}|\Omega| e^{d \lambda}\left|B_{d}\right| d^{-1},
$$

where $\left|B_{d}\right|$ is the measure of the unit ball in $\mathbb{R}^{d}$.

Proof Extending the eigenfunction $\varphi_{k}$ by zero outside $\Omega$ and using the Fourier transform we find

$$
\begin{aligned}
& \sum_{k}\left(\lambda-\lambda_{k}\right)_{+}=\sum_{k}\left(\lambda\left(\varphi_{k}, \varphi_{k}\right)-\left(\mathcal{H} \varphi_{k}, \varphi_{k}\right)\right)_{+} \\
& =\frac{1}{(2 \pi)^{d}}\left(\sum_{k} \int_{\mathbb{R}^{d}}(\lambda-\log (|\xi|))\left|\widehat{\varphi_{k}}(\xi)\right|^{2} d \xi\right)_{+} \\
& \leq \frac{1}{(2 \pi)^{d}} \int_{\mathbb{R}^{d}}(\lambda-\log (|\xi|))_{+} \sum_{k}\left|\widehat{\varphi_{k}}(\xi)\right|^{2} d \xi
\end{aligned}
$$

Using that $\left\{\varphi_{k}\right\}$ is an orthonormal basis in $L^{2}(\Omega)$ and denoting $e_{\xi}=e^{-i(\cdot, \xi)}$ we have

$$
\sum_{k}\left|\widehat{\varphi_{k}}(\xi)\right|^{2}=\sum_{k}\left|\left(e_{\xi}, \varphi_{k}\right)\right|^{2}=\left\|e_{\xi}\right\|_{L^{2}(\Omega)}^{2}=|\Omega|,
$$


and finally obtain

$$
\begin{aligned}
\sum_{k}\left(\lambda-\lambda_{k}\right)_{+} & \leq \frac{1}{(2 \pi)^{d}}|\Omega| \int_{\mathbb{R}^{d}}(\lambda-\log (|\xi|))_{+} \\
& =\frac{1}{(2 \pi)^{d}}|\Omega| e^{d \lambda} \int_{|\xi| \leq 1} \log \left(|\xi|^{-1}\right) d \xi
\end{aligned}
$$

We complete the proof by computing the last integral.

Let $\eta>\lambda$ and let us consider the function

$$
\psi_{\lambda}(t)=\frac{1}{\eta-\lambda}(\eta-t)_{+} .
$$

Denote by $\chi$ the step function

$$
\chi_{\lambda}(t)= \begin{cases}1, & \text { if } t<\lambda \\ 0, & \text { if } t \geq \lambda\end{cases}
$$

and let

$$
N(\lambda)=\#\left\{k: \lambda_{k}<\lambda\right\}
$$

be the number of the eigenvalues below $\lambda$ of the operator $\mathcal{H}$.

Then by using the previous statement we have

$$
N(\lambda) \leq \frac{1}{\eta-\lambda} \sum_{k}\left(\eta-\lambda_{k}\right)_{+} \leq \frac{1}{\eta-\lambda} \frac{1}{(2 \pi)^{d}}|\Omega| e^{d \eta}\left|B_{d}\right| d^{-1} .
$$

Minimising the right hand side w.r.t. $\eta$ we find $\eta=\lambda+\frac{1}{d}$ and thus obtain the following Corollary 3.2 For the number $N(\lambda)$ of the eigenvalues of the operator $\mathcal{H}$ below $\lambda$ we have

$$
N(\lambda) \leq e^{\lambda d+1} \frac{1}{(2 \pi)^{d}}|\Omega|\left|B_{d}\right| .
$$

\section{A lower bound for $\lambda_{1}(\Omega)$}

In this section, we focus on lower bounds for the first eigenvalue $\lambda_{1}=\lambda_{1}(\Omega)$. From Corollary 3.2, we readily deduce the following bound.

Theorem 4.1 Let $\Omega \subset \mathbb{R}^{d}$ be an open set of finite measure. Then we have

$$
\lambda_{1}(\Omega) \geq \frac{1}{d} \log \frac{(2 \pi)^{d}}{e|\Omega|\left|B_{d}\right|}
$$


In particular, if $|\Omega| \leq \frac{(2 \pi)^{d}}{e\left|B_{d}\right|}$, then the operator $\mathcal{H}$ does not have negative eigenvalues.

Proof If $\lambda<\frac{1}{d} \log \frac{(2 \pi)^{d}}{e|\Omega|\left|B_{d}\right|}$, then $N(\lambda)<1$ by (3.2), and therefore $N(\lambda)=0$. Consequently, $\mathcal{H}$ does not have eigenvalues below $\frac{1}{d} \log \frac{(2 \pi)^{d}}{e|\Omega|\left|B_{d}\right|}$.

Remark 1 Note that the inequalities (3.1), (3.2) and (4.1) hold for any open set $\Omega$ of finite measure without any additional conditions on its boundary.

In the following, we wish to improve the bound given in Theorem 4.1 in low dimensions $d$ for open boundary sets with Lipschitz boundary. We shall use the following Faber-Krahn type inequality.

Theorem 4.2 ([5, Corollary 1.6]) Let $\rho>0$. Among all bounded open sets $\Omega$ with Lipschitz, boundary and $|\Omega|=\rho$, the ball $B=B_{r}(0)$ with $|B|=\rho$ minimizes $\lambda_{1}(\Omega)$.

Corollary 4.3 For every open bounded sets $\Omega$ with Lipschitz boundary we have

$$
\lambda_{1}(\Omega) \geq \lambda_{1}\left(B_{d}\right)+\frac{1}{d} \log \frac{\left|B_{d}\right|}{|\Omega|},
$$

and equality holds if $\Omega$ is a ball.

Proof The result follows by combining Theorem 4.2 with the identity

$$
\lambda_{1}\left(B_{r}(0)\right)=\lambda_{1}\left(B_{d}\right)+\log \frac{1}{r} \quad \text { for } \quad r>0
$$

which follows from the scaling property of $\lambda_{1}$ noted in Lemma 2.5.

Corollary 4.3 gives a sharp lower bound, but it contains the unknown quantity $\lambda_{1}\left(B_{d}\right)$. By Theorem 4.1, we have

$$
\begin{aligned}
\lambda_{1}\left(B_{d}\right) & \geq \frac{1}{d} \log \frac{(2 \pi)^{d}}{e\left|B_{d}\right|^{2}}=\log (2 \pi)-\frac{1}{d}\left(1+2 \log \left|B_{d}\right|\right) \\
& =\frac{2}{d} \log \Gamma(d / 2)+\log 2+\frac{2}{d} \log \frac{d}{2}-\frac{1}{d} .
\end{aligned}
$$

The following theorem improves this lower bound in low dimensions $d \geq 2$.

Theorem 4.4 For $d \geq 2$, we have

$$
\lambda_{1}\left(B_{d}\right) \geq \log (2 \sqrt{d+2})-\frac{2^{d+1}\left|B_{d}\right|^{2}(d+2)^{\frac{d}{2}}}{d(2 \pi)^{2 d}} .
$$


Proof Let $u \in L^{2}\left(B_{d}\right)$ be radial with $\|u\|_{L^{2}}=1$. Then $\widehat{u}$ is also radial, and

$$
\begin{aligned}
|\widehat{u}(\xi)| & =|\widehat{u}(s)|=s^{1-\frac{d}{2}}\left|\int_{0}^{1} u(r) J_{\frac{d}{2}-1}(r s) r^{\frac{d}{2}} d r\right| \\
& \leq s^{1-\frac{d}{2}}\left(\int_{0}^{1} r^{d-1} u^{2}(r) d r\right)^{1 / 2}\left(\int_{0}^{1} r J_{\frac{d}{2}-1}^{2}(s r) d r\right)^{1 / 2} \\
& =\frac{s^{1-\frac{d}{2}}}{\sqrt{\left|S^{d-1}\right|}}\left(s^{-2} \int_{0}^{s} \tau J_{\frac{d}{2}-1}^{2}(\tau) d \tau\right)^{1 / 2} \\
& =\frac{s^{-\frac{d}{2}}}{\sqrt{\left|S^{d-1}\right|}}\left(\int_{0}^{s} \tau J_{\frac{d}{2}-1}^{2}(\tau) d \tau\right)^{1 / 2} \text { for } \xi \in \mathbb{R}^{d} \text { with } s=|\xi| .
\end{aligned}
$$

Consequently,

$$
\left|S^{d-1}\right||\widehat{u}(s)|^{2} \leq s^{-d} \int_{0}^{s} \tau J_{\frac{d}{2}-1}^{2}(\tau) d \tau
$$

In the case where, in addition, $u$ is a radial eigenfunction of (1.3) corresponding to $\lambda_{1}$ in $\Omega=B_{d}$, it follows that, for every $\lambda \in \mathbb{R}$,

$$
\begin{aligned}
& (2 \pi)^{d}\left[\lambda-\lambda_{1}\right]=\int_{\mathbb{R}^{d}}(\lambda-\ln |\xi|)|\widehat{u}(\xi)|^{2} d \xi \leq \int_{\mathbb{R}^{d}}(\lambda-\ln |\xi|)_{+}|\widehat{u}(\xi)|^{2} d \xi \\
& =\left|S^{d-1}\right| \int_{0}^{\infty} s^{d-1}(\lambda-\ln s)_{+}|\widehat{u}(s)|^{2} d s \leq \int_{0}^{\infty} \frac{(\lambda-\ln s)_{+}}{s} \int_{0}^{s} \tau J_{\frac{d}{2}-1}^{2}(\tau) d \tau d s \\
& =\int_{0}^{\infty} \tau J_{\frac{d}{2}-1}^{2}(\tau) \int_{\tau}^{\infty} \frac{(\lambda-\ln s)_{+}}{s} d s d \tau=\int_{0}^{e^{\lambda}} \tau J_{\frac{d}{2}-1}^{2}(\tau) \int_{\tau}^{e^{\lambda}} \frac{\lambda-\ln s}{s} d s d \tau \\
& =\int_{0}^{e^{\lambda}} \tau J_{\frac{d}{2}-1}^{2}(\tau) \int_{\ln \tau}^{\lambda}(\lambda-s) d s d \tau=\int_{0}^{e^{\lambda}} \tau J_{\frac{d}{2}-1}^{2}(\tau) \int_{0}^{\lambda-\ln \tau} s d s d \tau \\
& =\frac{1}{2} \int_{0}^{e^{\lambda}} \tau J_{\frac{d}{2}-1}^{2}(\tau)(\lambda-\ln \tau)^{2} d \tau=\frac{e^{2 \lambda}}{2} \int_{0}^{1} \tau J_{\frac{d}{2}-1}^{2}\left(e^{\lambda} \tau\right) \ln ^{2} \tau d \tau .
\end{aligned}
$$

We now use the following estimate for Bessel functions of the first kind:

$$
J_{v}(x) \leq \frac{x^{v}}{2^{v} \Gamma(v+1)} \text { for } \quad v>\sqrt{3}-2,0 \leq x<2 \sqrt{2(v+2)}
$$

A proof of this elementary estimate is given in the Appendix. We wish to apply (4.5) with $v=\frac{d}{2}-1$. This gives

$$
e^{2 \lambda} J_{\frac{d}{2}-1}^{2}\left(r_{0} e^{\lambda} \tau\right) \leq e^{d \lambda} \frac{\tau^{d-2}}{2^{d-2} \Gamma^{2}\left(\frac{d}{2}\right)}=\frac{d^{2}\left|B_{d}\right|^{2} e^{d \lambda}}{(2 \pi)^{d}} \tau^{d-2} \quad \text { for } \quad \tau \in[0,1]
$$


if $d \geq 2$ and $e^{\lambda} \leq 2 \sqrt{d+2}$, i.e., if

$$
d \geq 2 \text { and } \lambda \leq \log (2 \sqrt{d+2})
$$

Here we used that $\left|B_{d}\right|=\frac{2}{d} \frac{\pi^{\frac{d}{2}}}{\Gamma(d / 2)}$. Consequently, if (4.6) holds, we find that

$$
(2 \pi)^{d}\left[\lambda-\lambda_{1}\right] \leq \frac{d^{2}\left|B_{d}\right|^{2} e^{d \lambda}}{(2 \pi)^{d}} \int_{0}^{1} \tau^{d-1} \ln ^{2} \tau d \tau,
$$

where

$$
\int_{0}^{1} \tau^{d-1} \ln ^{2} \tau d \tau=-\frac{2}{d} \int_{0}^{1} \tau^{d-1} \ln \tau d \tau=\frac{2}{d^{2}} \int_{0}^{1} \tau^{d-1} d \tau=\frac{2}{d^{3}} .
$$

Hence

$$
(2 \pi)^{d}\left[\lambda-\lambda_{1}\right] \leq \frac{2\left|B_{d}\right|^{2}}{d(2 \pi)^{d}} e^{d \lambda}, \quad \text { i.e., } \quad \lambda_{1} \geq \lambda-\frac{2\left|B_{d}\right|^{2}}{d(2 \pi)^{2 d}} e^{d \lambda}
$$

Inserting the value $\lambda=\log (2 \sqrt{d+2})$ from (4.6), we deduce that

$$
\lambda_{1}=\lambda_{1}\left(B_{d}\right) \geq \log (2 \sqrt{d+2})-\frac{2^{d+1}\left|B_{d}\right|^{2}(d+2)^{\frac{d}{2}}}{d(2 \pi)^{2 d}},
$$

as claimed.

Remark 2 It seems instructive to compare the lower bounds given in (4.3) and (4.4) with other bounds obtained from spectral estimates which are already available in the literature. We first mention Beckner's logarithmic estimate of uncertainty [2, Theorem 1], which implies that ${ }^{1}$

$$
(\varphi, \varphi)_{\log } \geq \int_{\mathbb{R}^{d}}\left[\psi(d / 4)+\log \frac{2}{|x|}\right] \varphi^{2}(x) d x \geq[\psi(d / 4)+\log 2]\|\varphi\|_{2}^{2}
$$

for functions $\varphi \in C_{c}^{\infty}\left(B_{d}\right)$ and therefore

$$
\lambda_{1}\left(B_{d}\right) \geq \psi(d / 4)+\log 2
$$

Here, as before, $\psi=\frac{\Gamma^{\prime}}{\Gamma}$ denotes the Digamma function. Next we state a further lower bound for $(\varphi, \varphi)_{l o g}$ which follows from [5, Proposition 3.2 and Lemma 4.11]. We have

$$
(\varphi, \varphi)_{\log } \geq \zeta_{d}\|\varphi\|_{2}^{2} \quad \text { for } \varphi \in C_{c}^{\infty}\left(B_{d}\right)
$$

\footnotetext{
1 We note here that a different definition of Fourier transform is used in [2] and therefore the inequality looks slightly different
} 
where $\zeta_{d}$ is given in (2.7), i.e.,

$$
\zeta_{d}=\log 2+\frac{1}{2}(\psi(d / 2)-\gamma)=\left\{\begin{array}{c}
-\gamma+\sum_{k=1}^{\frac{d-1}{2}} \frac{1}{2 k-1}, \\
\log 2-\gamma+\sum_{k=1}^{\frac{d-2}{2}} \frac{1}{k},
\end{array} \quad d\right. \text { eden. }
$$

Inequality (4.8) implies that

$$
\lambda_{1}\left(B_{d}\right) \geq \zeta_{d}
$$

The latter inequality can also be derived from a lower bound of Bañuelos and Kulczycki for the first Dirichlet eigenvalue $\lambda_{1}^{\alpha}\left(B_{d}\right)$ of the fractional Laplacian $(-\Delta)^{\alpha / 2}$ in $B_{d}$. In [1, Corollary 2.2], it is proved that

$$
\lambda_{1}^{\alpha}\left(B_{d}\right) \geq 2^{\alpha} \frac{\Gamma\left(1+\frac{\alpha}{2}\right) \Gamma\left(\frac{d+\alpha}{2}\right)}{\Gamma\left(\frac{d}{2}\right)} \quad \text { for } \quad \alpha \in(0,2) .
$$

Combining this inequality with the characterization of $\lambda_{1}\left(B_{d}\right)$ given in [5, Theorem 1.5], we deduce that

$$
\lambda_{1}\left(B_{d}\right)=\lim _{\alpha \rightarrow 0^{+}} \frac{\lambda_{1}^{\alpha}\left(B_{d}\right)-1}{\alpha} \geq\left.\frac{d}{d \alpha}\right|_{\alpha=0} 2^{\alpha} \frac{\Gamma\left(1+\frac{\alpha}{2}\right) \Gamma\left(\frac{d+\alpha}{2}\right)}{\Gamma\left(\frac{d}{2}\right)}=\zeta_{d},
$$

as stated in (4.9).

We briefly comment on the quality of the lower bounds obtained here in low and high dimensions. In low dimensions $d \geq 2$, (4.4) is better than the bounds (4.3), (4.7) and (4.9). In dimension $d=1$ where the bound (4.4) is not available, the bound (4.3) yields the best value. The following table shows numerical values of the bounds $b_{1}(d)$, $b_{2}(d), b_{3}(d)$ resp. $b_{4}(d)$ given by (4.3), (4.4), (4.7), (4.9), respectively.

\begin{tabular}{lllllllllll}
\hline$d$ & 1 & 2 & 3 & 4 & 5 & 6 & 7 & 8 & 9 & 10 \\
\hline$b_{1}(d)$ & $-0,55$ & 0,19 & 0,55 & 0,79 & 0,97 & 1,12 & 1,25 & 1,36 & 1,46 & 1,55 \\
$b_{2}(d)$ & $/$ & 1,28 & 1,48 & 1,59 & 1,67 & 1,73 & 1,79 & 1,84 & 1,89 & 1,94 \\
$b_{3}(d)$ & -3.53 & $-1,27$ & $-0,39$ & 0,12 & 0,47 & 0,73 & 0,94 & 1,12 & 1,27 & 1,40 \\
$b_{4}(d)$ & $-0,58$ & 0,12 & 0,42 & 0,62 & 0,76 & 0,87 & 0,96 & 1,03 & 1,10 & 1,16 \\
\hline
\end{tabular}

To compare the bounds in high dimensions, we consider the asymptotics as $d \rightarrow \infty$. Since $\frac{\log \Gamma(t)}{t}=\log t-1+o(t)$ as $t \rightarrow \infty$, the bound (4.3) yields

$$
\lambda_{1}\left(B_{d}\right) \geq \log d-1+o(1) \quad \text { as } \quad d \rightarrow \infty,
$$


whereas (4.4) obviously gives

$$
\lambda_{1}\left(B_{d}\right) \geq \log \sqrt{d+2}+\log 2+o(1) \quad \text { as } \quad d \rightarrow \infty,
$$

Moreover, from (4.7) and the fact that

$$
\psi(t)=\log t+o(1) \quad \text { as } t \rightarrow \infty
$$

we deduce that

$$
\lambda_{1}\left(B_{d}\right) \geq \log d-\log 2+o(1) \quad \text { as } \quad d \rightarrow \infty
$$

Finally, (4.8) and (4.12) yield

$$
\lambda_{1}\left(B_{d}\right) \geq \log \sqrt{d}+\log 2-\frac{\gamma}{2}+o(1) \quad \text { as } \quad d \rightarrow \infty .
$$

So (4.13) provides the best asymptotic bound as $d \rightarrow \infty$.

Numerical computations indicate that the bound (4.4) is better than the other bounds for $2 \leq d \leq 21$, and (4.7) is the best among these bounds for $d \geq 22$.

\section{An asymptotic lower trace bound}

Throughout this section, we let $\Omega \subset \mathbb{R}^{d}$ denote an open set of finite measure. In this section we prove the following asymptotic lower bound. A similar statement was obtained in [9] for the Dirichlet boundary problem for a fractional Laplacian.

Theorem 5.1 For the eigenvalues of the problem (1.3) and any $\lambda \in \mathbb{R}$ we have

$$
\liminf _{\lambda \rightarrow \infty} e^{-d \lambda} \sum_{k}\left(\lambda-\lambda_{k}\right)_{+} \geq \frac{1}{(2 \pi)^{d}}|\Omega|\left|B_{d}\right| d^{-1} .
$$

Proof Let us fix $\delta>0$ and consider

$$
\Omega_{\delta}=\left\{x \in \Omega: \operatorname{dist}\left(x, \mathbb{R}^{d} \backslash \Omega\right)>\delta\right\} .
$$

Since $\delta$ is arbitrary it suffices to show the lower bound (5.1), where $\Omega$ is replaced by $\Omega_{\delta}$. Let $g \in C_{0}^{\infty}\left(\mathbb{R}^{d}\right)$ be a real-valued even function, $\|g\|_{L^{2}\left(\mathbb{R}^{d}\right)}=1$ with support in $\left\{x \in \mathbb{R}^{d}:|x| \leq \delta / 2\right\}$. For $\xi \in \mathbb{R}^{d}$ and $x \in \Omega_{\delta}$ we introduce the "coherent state"

$$
e_{\xi, y}(x)=e^{-i \xi x} g(x-y)
$$

Note that $\left\|e_{\xi, y}\right\|_{L^{2}\left(\mathbb{R}^{d}\right)}=1$. Using the properties of coherent states [15, Theorem 12.8] we obtain

$$
\sum_{k}\left(\lambda-\lambda_{k}\right)_{+} \geq \frac{1}{(2 \pi)^{d}} \int_{\mathbb{R}^{d}} \int_{\Omega_{\delta}}\left(e_{\xi, y},(\lambda-\mathcal{H})_{+} e_{\xi, y}\right)_{L^{2}(\Omega)} d y d \xi .
$$


Since $t \mapsto(\lambda-t)_{+}$is convex then applying Jensen's inequality to the spectral measure of $\mathcal{H}$ we obtain

$$
\sum_{k}\left(\lambda-\lambda_{k}\right)_{+} \geq \frac{1}{(2 \pi)^{d}} \int_{\mathbb{R}^{d}} \int_{\Omega_{\delta}}\left(\lambda-\left(\mathcal{H} e_{\xi, y}, e_{\xi, y}\right)_{L^{2}(\Omega)}\right)_{+} d y d \xi
$$

Next we consider the quadratic form

$$
\begin{aligned}
& \left(\mathcal{H} e_{\xi, y}, e_{\xi, y}\right)_{L^{2}(\Omega)}=\frac{1}{(2 \pi)^{d}} \int_{\mathbb{R}^{d}} \int_{\Omega} \int_{\Omega} e^{i(x-z)(\eta-\xi)} g(x-y) g(z-y) \log (|\eta|) d z d x d \eta \\
& =\frac{1}{(2 \pi)^{d}} \int_{\mathbb{R}^{d}} \int_{\Omega} \int_{\Omega} e^{i(x-z) \rho} g(x-y) g(z-y) \log (|\xi-\rho|) d z d x d \rho \\
& =\frac{1}{(2 \pi)^{d}} \int_{\mathbb{R}^{d}} \int_{\Omega} \int_{\Omega} e^{i(x-z) \rho} g(x-y) g(z-y)(\log |\xi|+\log (|\xi-\rho| /|\xi|)) d z d x d \rho \\
& =\log |\xi|+R(y, \xi)
\end{aligned}
$$

Since $g \in C_{0}^{\infty}\left(\mathbb{R}^{d}\right)$ we have for any $M>0$

$$
\begin{aligned}
& R(y, \xi) \\
& =\frac{1}{(2 \pi)^{d}} \int_{\mathbb{R}^{d}} \int_{\Omega} \int_{\Omega} e^{i(x-y) \rho} g(x-y) e^{i(y-z) \rho} g(z-y) \log (|\xi-\rho| /|\xi|) d z d x d \rho \\
& =\int_{\mathbb{R}^{d}}|\widehat{g}|^{2} \log (|\xi-\rho| /|\xi|) d \rho \leq C_{M} \int_{\mathbb{R}^{d}}(1+|\rho|)^{-M} \log (|\xi-\rho| /|\xi|) d \rho \\
& \leq C|\xi|^{-1} .
\end{aligned}
$$

Therefore from (5.2) we find

$$
\sum_{k}\left(\lambda-\lambda_{k}\right)_{+} \geq(2 \pi)^{-d}\left|\Omega_{\delta}\right| \int_{\mathbb{R}^{d}}\left(\lambda-\log |\xi|-C|\xi|^{-1}\right)_{+} d \xi
$$

Let us redefine the spectral parameter $\lambda=\ln \mu$. Then introducing polar coordinates we find

$$
\begin{aligned}
& \int_{\mathbb{R}^{d}}\left(\lambda-\log |\xi|-C|\xi|^{-1}\right)_{+} d \xi=\left|\mathbb{S}^{d-1}\right| \int_{0}^{\infty}\left(\ln \frac{\mu}{r}-\frac{C}{r}\right)_{+} r^{d-1} d r \\
& =\mu^{d}\left|\mathbb{S}^{d-1}\right| \int_{0}^{\infty}\left(\ln \frac{1}{r}-\frac{C}{\mu r}\right)_{+} r^{d-1} d r .
\end{aligned}
$$

The expression in the latter integral is positive if $-r \ln r>C \mu^{-1}$. The function $-r \ln r$ is concave. 


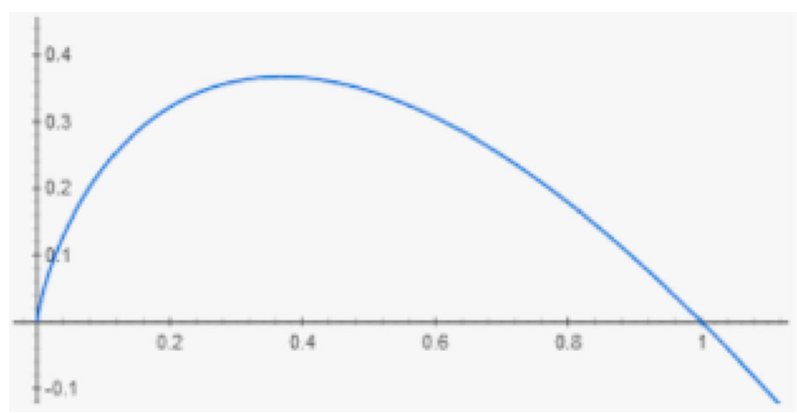

Its maximum is achieved at $r=1 / e$ at the value $1 / e$. The equation $-r \ln r=C \mu^{-1}$ has two solutions $r_{1}(\mu)$ and $r_{2}(\mu)$ such that $r_{1}(\mu) \rightarrow 0$ and $r_{2}(\mu) \rightarrow 1$ as $\mu \rightarrow \infty$. Therefore

$$
\begin{aligned}
& \int_{0}^{\infty}\left(\ln \frac{1}{r}-\frac{C}{\mu r}\right)_{+} r^{d-1} d r \geq \int_{r_{1}(\mu)}^{r_{2}(\mu)}\left(\ln \frac{1}{r}-\frac{C}{\mu r}\right) r^{d-1} d r \\
& =-\left.\frac{1}{d} r^{d} \ln r\right|_{r_{1}(\mu)} ^{r_{2}(\mu)}+\left.\frac{C}{\mu(d+1)} r^{d+1}\right|_{r_{1}(\mu)} ^{r_{2}(\mu)}+\left.\frac{1}{d^{2}} r^{d}\right|_{r_{1}(\mu)} ^{r_{2}(\mu)} \rightarrow \frac{1}{d^{2}} \quad \text { as } \quad \mu \rightarrow \infty
\end{aligned}
$$

Putting together (5.3), (5.4) and (5.5) and using $\mu=e^{\lambda}$ we obtain

$$
\liminf _{\lambda \rightarrow \infty} e^{-d \lambda} \sum_{k}\left(\lambda-\lambda_{k}\right)_{+} \geq \frac{1}{(2 \pi)^{d}}\left|\Omega_{\delta}\right|\left|B_{d}\right| d^{-1} .
$$

Since $\delta>0$ is arbitrary we complete the proof of Theorem 5.1.

\section{Weyl asymptotics}

Throughout this section, we let $\Omega \subset \mathbb{R}^{d}$ denote an open set of finite measure. Combining Theorems 3.1 and 5.1 we have

Theorem 6.1 The Riesz means of the eigenvalues of the Dirichlet boundary value problem (1.3) satisfy the following asymptotic formula

$$
\lim _{\lambda \rightarrow \infty} e^{-d \lambda} \sum_{k}\left(\lambda-\lambda_{k}\right)_{+}=\frac{1}{(2 \pi)^{d}}|\Omega|\left|B_{d}\right| d^{-1} .
$$

As a corollary we can obtain asymptotics of the number of the eigenvalues of the operator $\mathcal{H}$. 
Corollary 6.2 The number of the eigenvalues $N(\lambda)$ of the Dirichlet boundary value problem (1.3) below $\lambda$ satisfies the following asymptotic formula

$$
\lim _{\lambda \rightarrow \infty} e^{-d \lambda} N(\lambda)=\frac{1}{(2 \pi)^{d}}|\Omega|\left|B_{d}\right| .
$$

Proof In order to prove (6.2) we use two simple inequalities. If $h>0$, then

$$
\frac{\left(\lambda+h-\lambda_{k}\right)_{+}-\left(\lambda-\lambda_{k}\right)_{+}}{h} \geq 1_{(-\infty, \lambda)}\left(\lambda_{k}\right)
$$

and

$$
\frac{\left(\lambda-\lambda_{k}\right)_{+}-\left(\lambda-h-\lambda_{k}\right)_{+}}{h} \leq 1_{(-\infty, \lambda)}\left(\lambda_{k}\right)
$$

The inequality (6.3) implies, together with Theorems 3.1 and 5.1, that

$$
\begin{aligned}
& \limsup _{\lambda \rightarrow \infty} e^{-d \lambda} N(\lambda) \leq \limsup _{\lambda \rightarrow \infty} e^{-d \lambda} \sum_{k} \frac{\left(\lambda+h-\lambda_{k}\right)_{+}-\left(\lambda-\lambda_{k}\right)_{+}}{h} \\
& \leq \frac{1}{h}\left[e^{d h} \limsup _{\lambda \rightarrow \infty} e^{-d(\lambda+h)} \sum_{k}\left(\lambda+h-\lambda_{k}\right)_{+}-\liminf _{\lambda \rightarrow \infty} e^{-d \lambda} \sum_{k}\left(\lambda-\lambda_{k}\right)_{+}\right] \\
& \leq \frac{|\Omega|\left|B_{d}\right|}{d(2 \pi)^{d}} \frac{e^{d h}-1}{h} \quad \text { for every } h>0
\end{aligned}
$$

and thus

$$
\limsup _{\lambda \rightarrow \infty} e^{-d \lambda} N(\lambda) \leq \frac{|\Omega|\left|B_{d}\right|}{d(2 \pi)^{d}} \lim _{h \rightarrow 0^{+}} \frac{e^{d h}-1}{h}=\frac{|\Omega|\left|B_{d}\right|}{(2 \pi)^{d}} .
$$

Moreover, (6.3) implies, together with Theorems 3.1 and 5.1, that

$$
\begin{aligned}
& \liminf _{\lambda \rightarrow \infty} e^{-d \lambda} N(\lambda) \geq \liminf _{\lambda \rightarrow \infty} e^{-d \lambda} \sum_{k} \frac{\left(\lambda-\lambda_{k}\right)_{+}-\left(\lambda-h-\lambda_{k}\right)_{+}}{h} \\
& \geq \frac{1}{h}\left[e^{d h} \liminf _{\lambda \rightarrow \infty} e^{-d \lambda} \sum_{k}\left(\lambda-\lambda_{k}\right)_{+}-e^{-d h} \limsup _{\lambda \rightarrow \infty} e^{-d(\lambda-h)} \sum_{k}\left(\lambda-h-\lambda_{k}\right)_{+}\right] \\
& \geq \frac{|\Omega|\left|B_{d}\right|}{d(2 \pi)^{d}} \frac{1-e^{-d h}}{h} \quad \text { for every } h>0
\end{aligned}
$$

and therefore

$$
\liminf _{\lambda \rightarrow \infty} e^{-d \lambda} N(\lambda) \geq \frac{|\Omega|\left|B_{d}\right|}{d(2 \pi)^{d}} \lim _{h \rightarrow 0^{+}} \frac{1-e^{-d h}}{h}=\frac{|\Omega|\left|B_{d}\right|}{(2 \pi)^{d}} .
$$

The claim follows by combining (6.5) and (6.6).

Remark 3 The proof of Corollary 6.2 is a version of a Tauberian type arguments that is particularly simple due to properties of exponential functions. 


\section{An exact lower trace bound}

In this section we prove the following exact lower bound in the case of bounded open sets with Lipschitz boundary.

Theorem 7.1 Let $\Omega \subset \mathbb{R}^{d}, N \geq 2$ be an open bounded set with Lipschitz boundary, let $\tau \in(0,1)$, and let

$$
C_{\Omega, \tau}:=\frac{1}{|\Omega|(2 \pi)^{d}} \int_{\mathbb{R}^{d}}(1+|\rho|)^{\tau} \log (1+|\rho|)\left|\widehat{1_{\Omega}}(\rho)\right|^{2} d \rho,
$$

where $1_{\Omega}$ denotes the indicator function of $\Omega$.

For any $\lambda \geq 2 C_{\Omega, \tau}$, we have

$\sum_{k}\left(\lambda-\lambda_{k}\right)_{+} \geq \frac{|\Omega|\left|B_{d}\right|}{(2 \pi)^{d} d}\left[e^{d \lambda}-a_{\tau} C_{\Omega, \tau} e^{(d-\tau) \lambda}-b_{\tau} C_{\Omega, \tau}^{2} e^{(d-2 \tau) \lambda}-(d \lambda+1)\right]$

with $a_{\tau}:=\frac{d(d-\tau)-1}{d-\tau}$ and $b_{\tau}:=4 d \tau$.

Remark 4 In the definition of $C_{\Omega, \tau}$, we need $\tau<1$, otherwise the integral might not converge. In particular, if $\Omega=B_{d}$ is the unit ball in $\mathbb{R}^{d}$, we have

$$
\widehat{1_{\Omega}}(\rho)=(2 \pi)^{\frac{d}{2}}|\rho|^{-\frac{d}{2}} J_{\frac{d}{2}}(|\rho|)
$$

where $J_{\frac{d}{2}}(r)=O\left(\frac{1}{\sqrt{r}}\right)$ as $r \rightarrow \infty$. Hence the integral defining $C_{\Omega, \tau}$ converges if $\tau<1$. A similar conclusion arises for cubes or rectangles, where

$$
\widehat{1_{\Omega}}(\rho)=f_{1}\left(\rho_{1}\right) \cdots f_{d}\left(\rho_{d}\right)
$$

and $f_{j}(s)=O\left(\frac{1}{s}\right)$ as $|s| \rightarrow \infty, j=1, \ldots, d$.

On the other hand, if $\Omega \subset \mathbb{R}^{d}$ is an open bounded set with Lipschitz boundary, we have

$$
C_{\Omega, \tau}<\infty \quad \text { for } \tau \in(0,1) .
$$

Indeed, in this case, $\Omega$ has finite perimeter, i.e., $1_{\Omega} \in B V\left(\mathbb{R}^{d}\right)$. Therefore, as noted e.g. in [16, Theorem 2.14], $\Omega$ also has finite fractional perimeter

$$
P_{\tau}(\Omega)=\int_{\Omega} \int_{\mathbb{R}^{d} \backslash \Omega}|x-y|^{-d-\tau} d x d y=\frac{1}{2} \iint_{\mathbb{R}^{2 d}} \frac{\left(1_{\Omega}(x)-1_{\Omega}(y)\right)^{2}}{|x-y|^{d+\tau}} d x d y
$$

for every $\tau \in(0,1)$. Moreover, $P_{\tau}(\Omega)$ coincides, up to a constant, with the integral

$$
\int_{\mathbb{R}^{d}}|\rho|^{\tau}\left|\widehat{1_{\Omega}}(\rho)\right|^{2} d \rho
$$


which therefore is also finite for every $\tau \in(0,1)$. Since moreover $1_{\Omega}$ and therefore also $\widehat{1_{\Omega}}$ are functions in $L^{2}\left(\mathbb{R}^{d}\right)$ and for every $\varepsilon>0$ there exists $C_{\varepsilon}>0$ with

$$
(1+|\rho|)^{\tau} \log (1+|\rho|) \leq C_{\varepsilon}\left(1+|\rho|^{\tau+\varepsilon}\right) \quad \text { for } \rho \in \mathbb{R}^{d},
$$

it follows that (7.2) holds.

In the proof of Theorem 7.1, we will use the following elementary estimate.

Lemma 7.2 For $r \geq 0, s>0$ and $\tau \in(0,1)$, we have

$$
\log \left(1+\frac{r}{s}\right) \leq \frac{1}{s} \log (1+r) \quad \text { if } s \in(0,1)
$$

and

$$
\log \left(1+\frac{r}{s}\right) \leq \frac{(1+r)^{\tau}}{s^{\tau}} \log (1+r) \quad \text { if } s \geq 1
$$

In particular,

$$
\log \left(1+\frac{r}{s}\right) \leq \max \left\{\frac{1}{s}, \frac{1}{s^{\tau}}\right\}(1+r)^{\tau} \log (1+r) \quad \text { for } \quad r, s>0
$$

Remark 5 The obvious bound $\log \left(1+\frac{r}{s}\right) \leq \frac{r}{s}$ will not be enough for our purposes. We need an upper bound of the form $g(s) h(r)$ where $h$ grows less than linearly in $r$.

Proof of Lemma 7.2 Let first $s \in(0,1)$. Since

$$
\left.\log \left(1+\frac{r}{s}\right)\right|_{r=0}=0=\left.\frac{1}{s} \log (1+r)\right|_{r=0}
$$

and, for every $r>0$,

$$
\frac{d}{d r} \log \left(1+\frac{r}{s}\right)=\frac{1}{s+r} \leq \frac{1}{s+s r}=\frac{d}{d r} \frac{1}{s} \log (1+r),
$$

inequality (7.3) follows. To see (7.4), we fix $s>1$, and we note that

$$
\left.\log \left(1+\frac{r}{s}\right)\right|_{r=0}=0=\left.\frac{(1+r)^{\tau}}{s^{\tau}} \log (1+r)\right|_{r=0} .
$$

Moreover, for $0<r \leq s-1$, we have

$$
\begin{aligned}
& \frac{d}{d r} \frac{(1+r)^{\tau}}{s^{\tau}} \log (1+r)=\frac{(1+r)^{\tau-1}}{s^{\tau}}(1+\tau \log (1+r)) \\
& \quad \geq \frac{(1+r)^{\tau-1}}{s^{\tau}} \geq \frac{1}{s} \geq \frac{1}{s+r}=\frac{d}{d r} \log \left(1+\frac{r}{s}\right),
\end{aligned}
$$


so the inequality holds for $r \leq s-1$. If, on the other hand, $r \geq s-1$, we have obviously

$$
\log \left(1+\frac{r}{s}\right) \leq \log (1+r) \leq \frac{(1+r)^{\tau}}{s^{\tau}} \log (1+r)
$$

We may now complete the

Proof of Theorem 7.1 For $\xi \in \mathbb{R}^{d}$, we define $f_{\xi} \in L^{2}\left(\mathbb{R}^{d}\right)$ by $f_{\xi}(x)=\frac{1}{\sqrt{|\Omega|}} 1_{\Omega} e^{-i x \xi}$. Note that $\left\|f_{\xi}\right\|_{L^{2}\left(\mathbb{R}^{d}\right)}=1$ for any $\xi \in \mathbb{R}^{d}$. We write

$$
\begin{aligned}
\sum_{k}\left(\lambda-\lambda_{k}\right)_{+} & =\sum_{k}\left(\lambda-\lambda_{k}\right)_{+}\left\|\varphi_{k}\right\|_{L^{2}(\Omega)}^{2}=\frac{1}{(2 \pi)^{d}} \sum_{k}\left(\lambda-\lambda_{k}\right)_{+}\left\|\widehat{\varphi}_{k}\right\|_{L^{2}\left(\mathbb{R}^{d}\right)}^{2} \\
& =\frac{|\Omega|}{(2 \pi)^{d}} \sum_{k}\left(\lambda-\lambda_{k}\right)_{+} \int_{\mathbb{R}^{d}}\left|\left\langle f_{\xi}, \varphi_{k}\right\rangle\right|^{2} d \xi \\
& =\frac{|\Omega|}{(2 \pi)^{d}} \int_{\mathbb{R}^{d}} \sum_{k}\left(\lambda-\lambda_{k}\right)_{+}\left|\left\langle f_{\xi}, \varphi_{k}\right\rangle\right|^{2} d \xi
\end{aligned}
$$

Since $\sum_{k}\left|\left\langle f_{\xi}, \varphi_{k}\right\rangle\right|^{2}=\left\|f_{\xi}\right\|_{L^{2}\left(\mathbb{R}^{d}\right)}^{2}=1$ for $\xi \in \mathbb{R}^{d}$, Jensen's inequality gives

$$
\begin{aligned}
\sum_{k}\left(\lambda-\lambda_{k}\right)_{+} & \geq \frac{|\Omega|}{(2 \pi)^{d}} \int_{\mathbb{R}^{d}}\left(\lambda \sum_{k}\left|\left\langle f_{\xi}, \varphi_{k}\right\rangle\right|^{2}-\sum_{k} \lambda_{k}\left|\left\langle f_{\xi}, \varphi_{k}\right\rangle\right|^{2}\right)_{+} d \xi \\
& =\frac{|\Omega|}{(2 \pi)^{d}} \int_{\mathbb{R}^{d}}\left(\lambda-\sum_{k} \lambda_{k}\left|\left\langle f_{\xi}, \varphi_{k}\right\rangle\right|^{2}\right)_{+} d \xi \\
& =\frac{|\Omega|}{(2 \pi)^{d}} \int_{\mathbb{R}^{d}}\left(\lambda-\left(\mathcal{H} f_{\xi}, f_{\xi}\right)\right)_{+} d \xi
\end{aligned}
$$

Here, since

$$
\sqrt{|\Omega|} \widehat{f_{\xi}}(\eta-\xi)=\int_{\Omega} e^{-i(\eta-\xi) x} e^{-i x \xi} d x=\int_{\Omega} e^{-i \eta x} d x=\widehat{1_{\Omega}}(\eta)
$$

for $\eta, \xi \in \mathbb{R}^{d}$, we have

$$
\begin{aligned}
& |\Omega|(2 \pi)^{d}\left(\mathcal{H} f_{\xi}, f_{\xi}\right)=|\Omega| \int_{\mathbb{R}^{d}} \log |\eta|\left|\widehat{f_{\xi}}(\eta)\right|^{2} d \eta=|\Omega| \int_{\mathbb{R}^{d}} \log |\eta-\xi|\left|\widehat{f_{\xi}}(\eta-\xi)\right|^{2} d \eta \\
& \quad=\int_{\mathbb{R}^{d}} \log |\eta-\xi|\left|\widehat{1_{\Omega}}(\eta)\right|^{2} d \eta \leq \int_{\mathbb{R}^{d}}[\log |\xi|+\log (1+|\eta| /|\xi|)]\left|\widehat{1_{\Omega}}(\eta)\right|^{2} d \eta \\
& \quad \leq \log |\xi| \int_{\mathbb{R}^{d}}\left|\widehat{1_{\Omega}}(\eta)\right|^{2} d \eta+\max \left\{\frac{1}{|\xi|}, \frac{1}{|\xi|^{\tau}}\right\} \int_{\mathbb{R}^{d}}(1+|\eta|)^{\tau}\left(\log (1+|\eta|)\left|\widehat{1_{\Omega}}(\eta)\right|^{2} d \eta\right. \\
& \quad=|\Omega|(2 \pi)^{d}\left(\log |\xi|+\max \left\{\frac{1}{|\xi|}, \frac{1}{|\xi|^{\tau}}\right\} C_{\Omega, \tau}\right) \quad \text { for } \xi \in \mathbb{R}^{d},
\end{aligned}
$$


where $C_{\Omega, \tau}$ is defined in (7.1). Here we used Lemma 7.2. Combining (7.5) and (7.6), we get

$$
\sum_{k}\left(\lambda-\lambda_{k}\right)_{+} \geq \frac{|\Omega|}{(2 \pi)^{d}} \int_{\mathbb{R}^{d}}\left(\lambda-\log |\xi|-\max \left\{\frac{1}{|\xi|}, \frac{1}{|\xi|^{\tau}}\right\} C_{\Omega, \tau}\right)_{+} d \xi
$$

Let us redefine the spectral parameter $\lambda=\log \mu$ again. Then we find

$$
\begin{aligned}
& \int_{\mathbb{R}^{d}}\left(\lambda-\log |\xi|-\max \left\{\frac{1}{|\xi|}, \frac{1}{|\xi| \tau}\right\} C_{\Omega, \tau}\right)_{+} d \xi \\
& =\left|\mathbb{S}^{d-1}\right| \int_{0}^{\infty}\left(\log \frac{\mu}{r}-\max \left\{\frac{1}{r}, \frac{1}{r^{\tau}}\right\} C_{\Omega, \tau}\right)_{+} r^{d-1} d r \\
& =\mu^{d}\left|\mathbb{S}^{d-1}\right| \int_{0}^{\infty}\left(-\log r-\max \left\{\frac{1}{\mu^{1-\tau} r}, \frac{1}{r^{\tau}}\right\} \frac{C_{\Omega, \tau}}{\mu^{\tau}}\right)_{+} r^{d-1} d r \\
& \geq \mu^{d}\left|\mathbb{S}^{d-1}\right| \int_{\frac{1}{\mu}}^{\infty}\left(-\log r-\frac{1}{r^{\tau}} \frac{C_{\Omega, \tau}}{\mu^{\tau}}\right)_{+} r^{d-1} d r .
\end{aligned}
$$

For the last inequality, we used the fact that $\frac{1}{\mu^{1-\tau} r} \leq \frac{1}{r^{\tau}}$ for $r \geq \frac{1}{\mu}$.

Next we note that the function $r \mapsto f_{\mu}(r)=-\log r-\frac{1}{r^{\tau}} \frac{C_{\Omega, \tau}}{\mu^{\tau}}$ satisfies

$$
f_{\mu}(r)<0 \text { for } r \geq 1 \quad \text { and } \quad \lim _{r \rightarrow 0^{+}} f_{\mu}(r)=-\infty \text {. }
$$

Moreover, this function has two zeros $r_{1}(\mu), r_{2}(\mu)$ with $0<r_{1}(\mu)<\frac{1}{\mu}<r_{2}(\mu)<1$ and

$$
f_{\mu}(r) \geq 0 \quad \text { if and only if } \quad r_{1}(\mu) \leq r \leq r_{2}(\mu)
$$

To see this, we write

$$
f_{\mu}(r)=\frac{1}{r^{\tau}} g\left(r^{\tau}\right) \quad \text { with } \quad g(s)=-\frac{s}{\tau} \log s-\frac{C_{\Omega, \tau}}{\mu^{\tau}}
$$

and note that $g$ is strictly concave since $s \mapsto g^{\prime}(s)=-\frac{1}{\tau}-\log s$ is strictly decreasing. Consequently, $g$ has at most two zeros, and the same is true for $f$. Combining this with (7.9) and the fact that

$$
f(1 / \mu)=\log \mu-C_{\Omega, \tau}>0
$$

since $\lambda \geq 2 C_{\Omega, \tau}>C_{\Omega, \tau}$ by assumption, the claim above follows. From (7.8), we thus obtain the lower bound

$$
\int_{\mathbb{R}^{d}}\left(\lambda-\log |\xi|-\max \left\{\frac{1}{|\xi|}, \frac{1}{|\xi|^{\tau}}\right\} C_{\Omega, \tau}\right)_{+} d \xi
$$




$$
\geq \mu^{d}\left|\mathbb{S}^{d-1}\right| \int_{\frac{1}{\mu}}^{r_{2}(\mu)}\left(-\log r-\frac{1}{r^{\tau}} \frac{C_{\Omega, \tau}}{\mu^{\tau}}\right)_{+} r^{d-1} d r .
$$

Next, we claim that

$$
r_{2}(\mu) \geq r_{3}(\mu):=e^{\frac{1}{2 \tau}\left(\sqrt{1-\frac{4 \tau C_{\Omega, \tau}}{\mu^{\tau}}}-1\right)} .
$$

Here we note that $\frac{4 \tau C_{\Omega, \tau}}{\mu^{\tau}}=\frac{4 \tau C_{\Omega, \tau}}{e^{\tau \lambda}}<1$ since $\lambda \geq 2 C_{\Omega, \tau}$ by assumption. To see (7.11), we write

$$
r_{3}(\mu)=e^{-c_{\mu} \frac{C_{\Omega, \tau}}{\mu^{\tau}}} \quad \text { with } \quad c_{\mu}=\frac{\mu^{\tau}}{2 \tau C_{\Omega, \tau}}\left(1-\sqrt{1-\frac{4 \tau C_{\Omega, \tau}}{\mu^{\tau}}}\right),
$$

noting that

$$
\frac{\tau C_{\Omega, \tau}}{\mu^{\tau}} c_{\mu}^{2}-c_{\mu}+1=0
$$

and therefore

$$
\begin{aligned}
& f\left(r_{3}(\mu)\right)=f\left(e^{-\frac{c_{\mu} C_{\Omega, \tau}}{\mu^{\tau}}}\right)=\frac{c_{\mu} C_{\Omega, \tau}}{\mu^{\tau}}-\frac{1}{e^{-\tau \frac{c_{\mu} C_{\Omega, \tau}}{\mu^{\tau}}}} \frac{C_{\Omega, \tau}}{\mu^{\tau}} \\
& =\frac{C_{\Omega, \tau}}{\mu^{\tau} e^{-\tau \frac{c_{\mu} C_{\Omega, \tau}}{\mu^{\tau}}}}\left(c_{\mu} e^{-\tau \frac{c_{\mu} C_{\Omega, \tau}}{\mu^{\tau}}}-1\right) h \geq \frac{C_{\Omega, \tau}}{\mu^{\tau} e^{-\tau \frac{c_{\mu} C_{\Omega, \tau}}{\mu^{\tau}}}}\left(c_{\mu}\left(1-\tau \frac{c_{\mu} C_{\Omega, \tau}}{\mu^{\tau}}\right)-1\right)=0 .
\end{aligned}
$$

This proves (7.11). As a consequence of the inequality $\sqrt{1-a} \geq 1-\frac{a}{2}-\frac{a^{2}}{2}$ for $0 \leq a \leq 1$, we also have

$$
r_{3}(\mu) \geq e^{-\left(\frac{C_{\Omega, \tau}}{\mu^{\tau}}+\frac{4 \tau C_{\Omega, \tau}^{2}}{\mu^{2 \tau}}\right)}=: r_{4}(\mu)
$$

Consequently,

$$
\begin{aligned}
& \int_{\mathbb{R}^{d}}\left(\lambda-\log |\xi|-\max \left\{\frac{1}{|\xi|}, \frac{1}{|\xi|^{\tau}}\right\} C_{\Omega, \tau}\right)_{+} d \xi \\
& \geq \mu^{d}\left|\mathbb{S}^{d-1}\right| \int_{\frac{1}{\mu}}^{r_{4}(\mu)}\left(-\log r-\frac{1}{r^{\tau}} \frac{C_{\Omega, \tau}}{\mu^{\tau}}\right)_{+} r^{d-1} d r \\
& =\mu^{d}\left|\mathbb{S}^{d-1}\right|\left[-\frac{r^{d}}{d} \log r+\frac{1}{d^{2}} r^{d}-\frac{C_{\Omega, \tau}}{\mu^{\tau}(d-\tau)} r^{d-\tau}\right]_{\frac{1}{\mu}}^{r_{4}(\mu)} \\
& =\mu^{d}\left|\mathbb{S}^{d-1}\right|\left(\left[-\frac{r_{4}(\mu)^{d}}{d} \log r_{4}(\mu)+\frac{1}{d^{2}} r_{4}(\mu)^{d}-\frac{C_{\Omega, \tau}}{\mu^{\tau}(d-\tau)} r_{4}(\mu)^{d-\tau}\right]\right. \\
& \left.\quad-\left[\frac{\mu^{-d}}{d} \log \mu+\frac{1}{d^{2}} \mu^{-d}-\frac{C_{\Omega, \tau}}{\mu^{\tau}(d-\tau)} \mu^{\tau-d}\right]\right)
\end{aligned}
$$


which implies that

$$
\begin{aligned}
& \int_{\mathbb{R}^{d}}\left(\lambda-\log |\xi|-\max \left\{\frac{1}{|\xi|}, \frac{1}{|\xi|^{\tau}}\right\} C_{\Omega, \tau}\right)_{+} d \xi \\
& \geq \mu^{d}\left|\mathbb{S}^{d-1}\right|\left(\frac{1}{d^{2}} r_{4}(\mu)^{d}-\frac{C_{\Omega, \tau}}{\mu^{\tau}(d-\tau)} r_{4}(\mu)^{d-\tau}-\frac{\mu^{-d}}{d} \log \mu-\frac{1}{d^{2}} \mu^{-d}\right) \\
& =\mu^{d}\left|\mathbb{S}^{d-1}\right|\left(\frac{1}{d^{2}} e^{-d\left(\frac{C_{\Omega, \tau}}{\mu^{\tau}}+\frac{4 \tau C_{\Omega, \tau}^{2}}{\mu^{2 \tau}}\right)}-\frac{C_{\Omega, \tau}}{\mu^{\tau}(d-\tau)} e^{-(d-\tau)\left(\frac{C_{\Omega, \tau}}{\mu^{\tau}}+\frac{4 \tau C_{\Omega, \tau}^{2}}{\mu^{2 \tau}}\right)}\right. \\
& \left.\quad-\frac{\mu^{-d}}{d} \log \mu-\frac{1}{d^{2}} \mu^{-d}\right) .
\end{aligned}
$$

Since

$$
e^{-d\left(\frac{C_{\Omega, \tau}}{\mu^{\tau}}+\frac{{ }^{\tau} \tau C_{\Omega, \tau}^{2}}{\mu^{2 \tau}}\right)} \geq 1-d\left(\frac{C_{\Omega, \tau}}{\mu^{\tau}}+\frac{4 \tau C_{\Omega, \tau}^{2}}{\mu^{2 \tau}}\right)
$$

and

$$
e^{-(d-\tau)\left(\frac{C_{\Omega, \tau}}{\mu^{\tau}}+\frac{4 \tau C_{\Omega, \tau}^{2}}{\mu^{2 \tau}}\right)} \leq 1
$$

we conclude that

$$
\begin{aligned}
& \int_{\mathbb{R}^{d}}\left(\lambda-\log |\xi|-\max \left\{\frac{1}{|\xi|}, \frac{1}{|\xi|^{\tau}}\right\} C_{\Omega, \tau}\right)_{+} d \xi \\
& \geq \mu^{d} \frac{\left|\mathbb{S}^{d-1}\right|}{d^{2}}\left(1-d\left(\frac{C_{\Omega, \tau}}{\mu^{\tau}}+\frac{4 \tau C_{\Omega, \tau}^{2}}{\mu^{2 \tau}}\right)-\frac{C_{\Omega, \tau}}{\mu^{\tau}(d-\tau)}-\mu^{-d}(d \log \mu+1)\right) \\
& =\frac{\left|B_{d}\right|}{d}\left(\mu^{d}-C_{\Omega, \tau}\left(d-\frac{1}{d-\tau}\right) \mu^{d-\tau}-4 d \tau C_{\Omega, \tau}^{2} \mu^{d-2 \tau}-(d \log \mu+1)\right) \\
& =\frac{\left|B_{d}\right|}{d}\left(e^{d \lambda}-\frac{d(d-\tau)-1}{d-\tau} C_{\Omega, \tau} e^{(d-\tau) \lambda}-4 d \tau C_{\Omega, \tau}^{2} e^{(d-2 \tau) \lambda}-(d \lambda+1)\right)
\end{aligned}
$$

Combining the last estimate with (7.7), we get the asserted lower bound.

\section{Appendix: Note on a bound for Bessel functions}

The following elementary bound might be known but seems hard to find in this form.

Lemma 8.1 For $v \geq \sqrt{3}-2$ and $0 \leq x \leq 2 \sqrt{2(v+2)}$ we have

$$
\left|J_{v}(x)\right| \leq \frac{x^{v}}{2^{v} \Gamma(v+1)}
$$


Proof We use the representation

$$
J_{v}(x)=\left(\frac{x}{2}\right)^{v} \sum_{m=0}^{\infty} \frac{(-1)^{m}}{m ! \Gamma(m+v+1)}\left(\frac{x}{2}\right)^{2 m} .
$$

For $0 \leq x \leq 2 \sqrt{2(v+2)}$ and $m \geq 1$, we have

$$
\left(\frac{x}{2}\right)^{2} \leq(m+1)(m+v+1)=\frac{(m+1) \Gamma(m+v+2)}{\Gamma(m+v+1)}
$$

and therefore

$$
\frac{\Gamma(v+1)}{(m+1) ! \Gamma(m+v+2)}\left(\frac{x}{2}\right)^{2(m+1)} \leq \frac{\Gamma(v+1)}{m ! \Gamma(m+v+1)}\left(\frac{x}{2}\right)^{2 m} .
$$

Consequently,

$$
J_{v}(x)=\frac{x^{v}}{2^{v} \Gamma(v+1)}\left[1+\sum_{m=1}^{\infty} \frac{(-1)^{m} \Gamma(v+1)}{m ! \Gamma(m+v+1)}\left(\frac{x}{2}\right)^{2 m}\right] \leq \frac{x^{v}}{2^{v} \Gamma(v+1)} .
$$

From (8.1) we also deduce that

$$
\begin{aligned}
J_{v}(x) & \geq \frac{x^{v}}{2^{v} \Gamma(v+1)}\left[1-\frac{\Gamma(v+1)}{\Gamma(v+2)}\left(\frac{x}{2}\right)^{2}+\frac{\Gamma(v+1)}{2 \Gamma(v+3)}\left(\frac{x}{2}\right)^{4}-\frac{\Gamma(v+1)}{6 \Gamma(v+4)}\left(\frac{x}{2}\right)^{6}\right] \\
& =\frac{x^{v}}{2^{v} \Gamma(v+1)}\left[1-\frac{1}{v+1} f\left(\left(\frac{x}{2}\right)^{2}\right)\right]
\end{aligned}
$$

with $f: \mathbb{R} \rightarrow \mathbb{R}$ given by $f(t)=t-\frac{t^{2}}{2(v+2)}+\frac{t^{3}}{6(v+2)(v+3)}$. Since

$$
f^{\prime}(t)=1-\frac{t}{v+2}+\frac{t^{2}}{2(v+2)(v+3)}, \quad \text { and } \quad f^{\prime \prime}(t)=\frac{1}{v+2}\left(\frac{t}{v+3}-1\right)
$$

we have

$f^{\prime}(t) \geq f^{\prime}(v+3)=1-\frac{v+3}{v+2}+\frac{v+3}{2(v+2)}=1-\frac{1}{2} \frac{v+3}{v+2} \geq 0$ for $t \in \mathbb{R}$ if $v \geq-1$

and therefore

$$
f(t) \leq f(2(v+2))=2(v+2)-\frac{[2(v+2)]^{2}}{2(v+2)}+\frac{[2(v+2)]^{3}}{6(v+2)(v+3)}=\frac{4(v+2)^{2}}{3(v+3)}
$$

for $t \leq 2(v+2)$ if $v \geq-1$. Since $\frac{4(v+2)^{2}}{3(v+3)} \leq \frac{2}{v+1}$ for $v \geq \sqrt{3}-2$, we conclude that

$$
J_{v}(x) \geq \frac{x^{v}}{2^{v} \Gamma(v+1)}\left[1-\frac{1}{v+1} f\left(\left(\frac{x}{2}\right)^{2}\right)\right] \geq-\frac{x^{v}}{2^{v} \Gamma(v+1)} .
$$


for $v \geq \sqrt{3}-2$ and $0 \leq x \leq 2 \sqrt{2(v+2)}$. The claim thus follows.

Data Availability The data availability statement is not included in the paper.

\section{Compliance with ethical standards}

Conflict of interest The authors declare that there is no conflict of interest.

Open Access This article is licensed under a Creative Commons Attribution 4.0 International License, which permits use, sharing, adaptation, distribution and reproduction in any medium or format, as long as you give appropriate credit to the original author(s) and the source, provide a link to the Creative Commons licence, and indicate if changes were made. The images or other third party material in this article are included in the article's Creative Commons licence, unless indicated otherwise in a credit line to the material. If material is not included in the article's Creative Commons licence and your intended use is not permitted by statutory regulation or exceeds the permitted use, you will need to obtain permission directly from the copyright holder. To view a copy of this licence, visit http://creativecommons.org/licenses/by/4.0/.

\section{References}

1. Bañuelos, R., Kulczycki, T.: The Cauchy process and the Steklov problem. J. Funct. Anal. 211, 355-423 (2004)

2. Beckner, W.: Pitt's inequality and the uncertainty principle. Proc. AMS 123(6), 1897-1905 (1995)

3. Berezin, F.A.: Convex functions of operators. Mat. Sb. 88, 268-278 (1972)

4. Berezin, F.A.: Covariant and contravariant symbols of operators. English transl. Math. USSR Izvestija 6, 1117-1151 (1972)

5. Chen, H., Weth, T.: The Dirichlet problem for the logarithmic Laplacian. Comm. Partial Diff. Equ. 44, 1100-1139 (2019)

6. De Luca, L., Novaga, M., Ponsiglione, M.: The 0-fractional perimeter between fractional perimeters and Riesz potentials, Preprint, arXiv:1906.06303

7. Felsinger, M., Kassmann, M., Voigt, P.: The Dirichlet problem for nonlocal operators. Math. Zeit. 279, 779-809 (2015)

8. Feulefack, P. A., Jarohs, S., Weth, T.: Small order asymptotics of the Dirichlet eigenvalue problem for the fractional Laplacian, Preprint, arXiv:2010.10448

9. Geisinger, G.: A short proof of Weyl's law for fractional operators. JMP 55, 0011504 (2014). https:// doi.org/10.1063/1.4861935

10. Jarohs, S., Weth, T.: Local compactness and nonvanishing for weakly singular nonlocal quadratic forms. Nonlinear Anal. 193, 111431 (2020)

11. Jarohs, S., Saldaña, A., Weth, T.: A new look at the fractional Poisson problem via the logarithmic Laplacian. J. Funct. Anal. (2020). https://doi.org/10.1016/j.jfa.2020.108732

12. Laptev, A.: Dirichlet and Neumann eigenvalue problems on domains in euclidean spaces. J. Funct. Anal. 151, 531-545 (1997)

13. Li, P., Yau, S.-T.: On the Schrödinger equation and the eigenvalue problem. Comm. Math. Phys. 88, 309-318 (1983)

14. Lieb, E.H.: The classical limit of quantum spin systems. Comm. Math. Phys. 31, 327-340 (1973)

15. E.H. Lieb and M. Loss, Analysis, AMS, Graduate Studies in Mathematics, 14, 1997, 346 pages

16. Lombardini, L.: Fractional Perimeter and Nonlocal Minimal Surfaces, arxiv:1508.06241

17. Musina, R., Nazarov, A.I.: On fractional Laplacians. Comm. Part. Diff. Eq. 39, 1780-1790 (2014)

18. Pego, R.L.: Compactness in $L^{2}$ and the Fourier transform. Proc. Amer. Math. Soc. 95, 252-254 (1985)

19. Rozenblum, G.V.: Distribution of the discrete spectrum of singular differential operators. Dokl. AN SSSR 202, 1012-1015 (1972)

20. Rozenblum, G.V.: Distribution of the discrete spectrum of singular differential operators. Izv. Vuzov, Matematika 1, 75-86 (1976)

Publisher's Note Springer Nature remains neutral with regard to jurisdictional claims in published maps and institutional affiliations. 